\section{Evidências do impacto da suplementação múltipla com micronutrientes no crescimento de pré-escolares: revisão sistemática}

\section{Evidence of the impact of multiple micronutrient supplements on the growth of pre-school children: a systematic review}

Dixis Figueroa Pedraza 1 1 Programa de Pós-graduação em Saúde Pública. Universidade
Estadual da Paraíba. Av. das Baraúnas, 351. Campus Universitário.
Bairro Bodocongó. Campina Grande, PB, Brasil. CEP: 58.109-753. E-mail: dixisfigueroa@gmail.com

\begin{abstract}
Objectives: to put together evidence of the effect of multiple micronutrient supplements on the growth of pre-school children, with special emphasis on the effects in combination or in isolation of zinc, iron and vitamin $A$.

Methods: the PubMed database was searched for randomized clinical trials and meta-analytical systematic review articles. The selected articles were published between 1995 and May 2013, using a range of descriptors (child, preschool OR infant) AND (growth) AND (zinc AND iron AND vitamin A) OR (zinc $A N D$ iron) OR (zinc $A N D$ vitamin A) OR (iron AND vitamin A).

Results: thirty-three articles were selected for the present review. Twenty-nine were clinical trials and four meta-analytical systematic reviews. The administration of nutritional supplements containing combinations of zinc, iron and vitamin A may increase the potential for growth in children and has a stronger effect than multiple supplements, a placebo or the child's habitual diet, or than single or dual supplements. The combination of zinc and vitamin $A$ is the best dual supplement option. The effect of the three nutrient supplement compared with the other options (placebo, single, dual, or multiple supplements), as well as the various effects of supplementation according to the nutritional status and/or age of the children, still require more in-depth investigation to provide a guide for prevention strategies.

Conclusions: multiple micronutrient supplements have positive effects on linear growth the extent of which depend on the supplementation regimen adopted.
\end{abstract}

Key words Growth, Body height, Micronutrients, Zinc, Iron, Vitamin A, Child, preschool

\section{Resumo}

Objetivos: agregar evidências sobre o efeito da suplementação múltipla com micronutrientes no crescimento de crianças pré-escolares, com ênfase nos efeitos combinados ou isolados do zinco, ferro e vitamina $A$.

Métodos: foi realizada uma busca por ensaios clínicos aleatorizados e artigos de revisão sistemática com metanálise na base de dados PubMed. Foram selecionados artigos publicados entre 1995 e maio de 2013, considerando a combinação dos descritores (child, preschool OR infant) AND (growth) AND (zinc $A N D$ iron $A N D$ vitamin $A$ ) OR (zinc $A N D$ iron) $O R$ (zinc $A N D$ vitamin $A$ ) OR (iron AND vitamin A).

Resultados: foram selecionados 33 artigos para a presente revisão, 29 do tipo ensaio clínico e quatro de revisão sistemática com metanálise. A administração de suplementos nutricionais contendo combinações de zinco, ferro e vitamina A pode aumentar o potencial de crescimento das crianças, sendo mais evidente o efeito quando comparada a suplementação com múltiplos micronutrientes vs placebo ou dieta habitual, e com os esquemas de suplementação dupla e única. A combinação de zinco e vitamina $A$ é a melhor opção de suplementação dupla. O efeito da suplementação tripla em relação às outras opções (placebo, única, dupla, múltipla), bem como os possiveis efeitos diferenciados da suplementação segundo a condição nutricional elou idade das crianças, ainda precisam de investigação mais aprofundada para orientar as estratégias de prevenção.

Conclusões: a suplementação múltipla com micronutrientes tem efeitos positivos no crescimento linear que dependem do esquema de suplementação adotado.

Palavras-chave Crescimento, Estatura, Micronutrientes, Zinco, Ferro, Vitamina A, Préescolar

Rev. Bras. Saúde Matern. Infant., Recife, 14 (1): 17-37 jan. / mar., 2014 


\section{Introdução}

Nas sociedades modernas, as crianças constituem um dos grupos populacionais mais vulneráveis em relação ao desenvolvimento de carências nutricionais. ${ }^{1}$ Sabe-se que o potencial genético de crescimento pode ser ou não alcançado dependendo das condições de vida a que o indivíduo esteja exposto, desde a concepção até a idade adulta, tornando-se evidente a influência dos fatores extrínsecos no crescimento físico. ${ }^{2} \mathrm{O}$ crescimento é, assim, influenciado pela interação das características genéticas e a disponibilidade de macro e micronutrientes. $3 \mathrm{O}$ zinco ( $\mathrm{Zn}$ ), o ferro (Fe) e a vitamina A (vit. A) são os micronutrientes mais importantes no crescimento infantil, suas deficiências apresentam altas prevalências em países em desenvolvimento tendo como principal causa a redução de conteúdo e da biodisponibilidade na dieta. 4

$\mathrm{O}$ zinco influencia a regulação hormonal da divisão celular, especialmente via hormônio do crescimento $(\mathrm{GH})$ e fator I do crescimento dependente de insulina (IGF-I), além de interferir em hormônios mitogênicos, atuando sobre a proliferação celular. $5 \mathrm{O}$ ferro é o oligoelemento mais abundante no organismo humano e participa de diferentes processos metabólicos, incluindo o transporte de elétrons, metabolismo de catecolaminas (co-fator da enzima tirosina hidroxilase) e síntese de DNA.6 Além disso, o ferro é componente de estruturas essenciais ao funcionamento fisiológico, a exemplo da hemoglobina na qual atua no transporte de oxigênio até os tecidos. ${ }^{3} \mathrm{~A}$ vit. A atua na manutenção do tecido epitelial, síntese proteica, diferenciação de células ósseas, desenvolvimento do osso e secreção noturna do $\mathrm{GH}$, bem como no adequado funcionamento do sistema imunológico.3,6

$\mathrm{O}$ zinco, o ferro e a vit. A apresentam importantes interações, tanto em termos funcionais quanto de absorção. ${ }^{7}$ A deficiência de tais nutrientes pode ocorrer de forma isolada ou concomitante, possuindo efeitos independentes ou em interação sobre a saúde e o crescimento do indivíduo. ${ }^{4,8}$ Discute-se se a suplementação com um único micronutriente teria efeito significativo nos casos da coexistência das deficiências de micronutrientes e se constituiria a estratégia mais custo-efetiva para problemas de crescimento associados a um micronutriente específico. 4,9 Vários estudos têm indicado que o efeito da suplementação com micronutrientes depende do estado nutricional prévio das crianças, dentre outras características como a idade, o estado de saúde/desenvolvimento de doenças infecciosas e diferenças ambientais. $4,10,11$
A atenção quanto ao impacto das intervenções com micronutrientes decorre não somente da sua importância como medida preventiva, como também de preservação do bom estado nutricional, de saúde e da capacidade cognitiva da criança. ${ }^{11} \mathrm{O}$ déficit de estatura está associado à ocorrência de doenças crônicas, prejuízos na oxidação de gorduras e intolerância à glicose, bem como contribui para a baixa estatura na idade adulta. Um adulto com déficit estatural pode apresentar limitações funcionais que podem reduzir sua capacidade de trabalho e, consequentemente, afetar a sua saúde e de seus dependentes. Mulheres com baixa estatura tendem a gerar uma criança com as mesmas condições, formando um ciclo vicioso de consequências negativas. ${ }^{12}$

Considerando a relevância do tema, o objetivo deste estudo foi agregar evidências sobre o efeito da suplementação múltipla com micronutrientes no crescimento de crianças pré-escolares, com ênfase nos efeitos combinados ou isolados do zinco, ferro e vit. A.

\section{Métodos}

Foi realizada uma busca sistemática por ensaios clínicos aleatorizados e estudos de revisão sistemática com metanálise que analisaram o impacto da suplementação com dois ou mais micronutrientes no crescimento de crianças préescolares. Foram incluídos estudos que envolveram a avaliação de intervenções com zinco, ferro e vit. A. A escolha desses delineamentos se deveu à escassez de estudos de sistematização do efeito da suplementação com múltiplos micronutrientes no crescimento linear, que tem contribuído para as incertezas que ainda persistem sobre o impacto da suplementação múltipla com micronutrientes no crescimento; as importantes interações entre diferentes micronutrientes e às controvérsias relacionadas ao custo-efetividade da suplementação múltipla com micronutrientes em relação a outras medidas de intervenção.

As buscas foram realizadas na base de dados PubMed (National Library of Medicine, Bethesda, MD) em 23 de maio de 2013, considerando artigos publicados a partir de 01/01/1995. Como estratégia de busca na obtenção dos estudos foi utilizada a combinação dos descritores (child, preschool OR infant) AND (growth) AND (zinc AND iron AND vitamin A) OR (zinc AND iron) OR (zinc AND vitamin A) OR (iron AND vitamin A). Para os ensaios clínicos aleatorizados, a busca limitou-se à opção clinical trial e para os estudos de revisão sistemática com metanálise à opção review. Foram 
utilizadas as ferramentas próprias do PubMed de limites de pesquisa por intervalo temporal e tipo de delineamento de estudo.

Inicialmente procedeu-se a identificação e eliminação dos artigos duplicados decorrentes do uso das diferentes estratégias de busca e diversidade de descritores.

Realizou-se igualmente consulta às listas de referências dos estudos que obedeceram aos critérios de inclusão para a localização de artigos que não tivessem sido identificados na busca inicial.

O processo de seleção dos estudos envolveu duas etapas: a primeira compreendeu a leitura dos títulos e dos resumos e a seleção daqueles considerados adequados de acordo com critérios de inclusão, segundo o tipo de estudo (ensaios clínicos aleatorizados ou estudos de revisão sistemática com metanálise) e participantes (crianças pré-escolares).
Em seguida, realizou-se a leitura da seção metodologia dos estudos que na fase anterior foram considerados adequados, analisando-os segundo o tipo de intervenção e desfechos estudados (Tabela 1). Os artigos selecionados a partir das listas de referências foram igualmente selecionados com base nos critérios de inclusão e exclusão.

Os ensaios clínicos aleatorizados foram caracterizados considerando autor e ano de publicação, país de realização do estudo, grupos de estudo e duração da suplementação, características dos participantes e amostra, de estudo, medidas de efeito analisadas e principais resultados (efeitos da intervenção). Os artigos de revisão sistemática com metanálise foram caracterizados segundo autor e ano de publicação, número de estudos incluídos na revisão, estratégias de suplementação avaliadas e impacto observado.

Tabela 1

Critérios para a inclusão e exclusão dos estudos controlados aleatorizados na revisão sobre o efeito da suplementação múltipla com micronutrientes no crescimento de crianças pré-escolares.

Parâmetros Critérios de inclusão Critérios de exclusão

Tipos de estudos

- ensaios clínicos aleatorizadosa - estudos de revisão sistemática com metanálisea

Participantes - crianças pré-escolaresa

- estudo não experimental

- revisão sistemática sem metanálise

- outro estudo diferente de revisão

- idade inapropriada (crianças não préescolares, adolescentes, adultos, gestantes)

- crianças com patologias que podem afetar o crescimento

Tipos de intervenção*

Desfechos clínicos

\begin{abstract}
- suplementação com no mínimo dois dos seguintes micronutrientes: zinco, ferro, vit. A b

- suplementação com dois ou mais micronutrientes versus placebo ou suplementação com um único micronutriente $b$

- suplementação com dois ou mais micronutrientes versus dieta habitualb
\end{abstract}

- crescimento: ganho de peso, ganho de estatura, Z-escores de E/I, Zescores de $\mathrm{P} / \mathrm{E}$, Z-escores de $\mathrm{P} / \mathrm{I}$ b

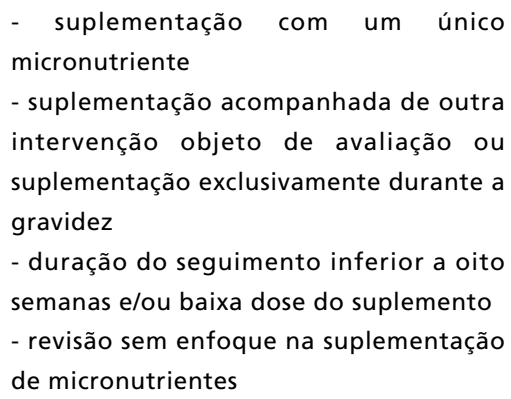

- sem análise do efeito da suplementação no crescimento

\footnotetext{
* Considerou-se suplementação tanto as intervenções através de alimentos fortificados com micronutrientes quanto através de suplementos medicamentosos; a Identificados através da leitura de títulos e resumos dos artigos; b Identificados através da leitura da metodologia dos artigos.
} 
Classificação dos estudos e análise do impacto da suplementação múltipla com micronutrientes

Os ensaios clínicos aleatórios foram agrupados em seis categorias de estudo segundo o tipo de intervenção e grupos de comparação: a) suplementação com múltiplos micronutrientes $v s$ dieta habitual ou placebo; b) suplementação tripla ou dupla $v s$ placebo; c) suplementação com múltiplos micronutrientes $v s$ suplementação tripla, dupla ou única; d) suplementação tripla vs suplementação dupla ou única; e) suplementação dupla vs suplementação única.

Considerou-se suplementação com múltiplos micronutrientes aquela que incluía mais de cinco micronutrientes, entre os quais zinco, ferro e vit. A; suplementação tripla, a que incluía zinco, ferro e vit. $\mathrm{A}$, acompanhada ou não por outro(s) nutriente(s) com os quais apresentam interação (vitamina $\mathrm{C}$, ácido fólico, vitamina $\mathrm{D}$, cálcio); suplementação dupla, a que continha zinco e ferro ou zinco e vit. A ou ferro e vit. A, acompanhada ou não por outro(s) nutriente(s) com os quais apresentam interação (vit $\mathrm{C}$, ácido fólico, vit. $\mathrm{D}$, cálcio); e suplementação única, a que continha zinco ou ferro ou vit. A, acompanhada ou não por outro(s) nutriente(s) com o(s) qual(is) apresenta(m) interação (vit. C, ácido fólico, vit. $\mathrm{D}$, cálcio).

Os efeitos da suplementação múltipla com micronutrientes demonstrados nos ensaios clínicos aleatórios foram interpretados considerando-se o delineamento dos ensaios, as intervenções e as características dos participantes.

Os resultados dos estudos de revisão sistemática com metanálise foram descritos e interpretados utilizando a mesma estratégia utilizada para a interpretação dos resultados dos ensaios clínicos aleatórios.

O impacto da suplementação múltipla foi analisado sobre três dimensões do crescimento: a) crescimento linear (ganho de estatura e ganho de Z-escores de estatura/idade); b) crescimento ponderal (ganho de peso e ganho de Z-escores de peso/estatura); c) crescimento global (ganho de Z-escores de peso/idade)

\section{Resultados}

Inicialmente foram identificados 110 ensaios clínicos aleatorizados e 74 estudos de revisão sistemática com metanálise. Após análise dos critérios de inclusão e exclusão, foram considerados elegíveis 26 artigos do tipo ensaio clínico e três de revisão sistemática com metanálise. Abusca por artigos nas listas de referências dos estudos selecionados para a revisão possibilitou a identificação e inclusão de três ensaios clínicos e um artigo de revisão sistemática com metanálise. O fluxo relacionado à identificação, exclusão e inclusão dos estudos encontra-se na Tabela 2.

\section{Ensaios clínicos aleatorizados}

As características dos ensaios clínicos aleatórios incluídos na revisão13-41 são apresentadas nas Tabelas 3 e 4. A intervenção por meio da fortificação de alimentos foi utilizada em oito estudos $13-20$ (Tabela 3) enquanto a suplementação medicamentosa foi utilizada em 21.21-41 (Tabela 4). Vários autores utilizaram algum critério de risco nutricional25,34-36,39 ou de vulnerabilidade geográfica14,15,17-19,22,24,26,27,29,31,34,36-38,41 para a seleção da população de estudo. Observou-se igualmente variações na duração da intervenção, sendo a suplementação por seis meses (18 estudos),15-18,20,21,23,24,26,27,29-33,36,37,40 a mais frequente, seguida pela suplementação por 12 meses (cinco estudos). $14,28,35,38,41 \quad$ Em quatro estudos 13,19,34,39 a intervenção foi inferior a seis meses e em dois, 22,25 superior a 12 meses.

Do total de 29 artigos, o ganho de peso foi usado como medida de efeito em 22,13,14,16,1824,26,27,29,31,32,35-41 o Z-escore de peso/estatura em $23,13-18,20,21-24,26-29,31-34,37-39,41$ o ganho de estatura em $2213,14,16,18-24,26,27,29,31,32,35-41$ e os Z-escore de estatura/idade e peso/idade em 27.13-18,20,21-34,36-41 De acordo com a classificação adotada, a suplementação com múltiplos micronutrientes vs dieta habitual ou placebo foi investigada em 11 estudos,14,16-20,29-32,38 a suplementação tripla ou dupla $v s$ placebo em 13 estudos,23-28,33,35-37,39-41 a suplementação com múltiplos micronutrientes $v s$ suplementação tripla, dupla ou única em sete estudos, $15,21,22,29-32$ a suplementação tripla $v s$ suplementação dupla ou única em dois estudos 24,28 e a suplementação dupla vs suplementação única em 14 estudos. 15,21,23-27,33-37,40,41 O esquema de suplementação testado por Samadpour et al. 13 não se adequou a nenhuma das seis categorias de intervenção.

Os efeitos dos diferentes tipos de suplementação sobre o crescimento linear, ponderal e global verificados nos ensaios clínicos selecionados são apresentados a seguir.

\section{I - Suplementação com múltiplos micronu-}

trientes vs dieta habitual ou placebo

De um total de 11 estudos identificados 14,16-20,2932,38 , quatro $14,19,30,38$ mostraram efeito positivo sobre o crescimento linear, sendo três $19,30,38$ em 
Estudos identificados, excluídos e incluídos na revisão sobre o efeito da suplementação múltipla com micronutrientes no crescimento de crianças pré-escolares.

\begin{tabular}{|c|c|c|c|}
\hline \multirow[b]{2}{*}{ Etapas } & \multirow[b]{2}{*}{ Identificação, exclusão e inclusão dos estudos } & \multicolumn{2}{|c|}{ Número de artigos } \\
\hline & & Ensaio clínico & $\begin{array}{l}\text { Revisão com } \\
\text { metanálise }\end{array}$ \\
\hline \multirow[t]{7}{*}{1} & Estudos identificados (descritores de micronutrientes) & & \\
\hline & - zinc AND iron AND vitamin A & 26 & 20 \\
\hline & - zinc AND iron & 87 & 57 \\
\hline & - zinc AND vitamin A & 39 & 26 \\
\hline & - iron AND vitamin A & 36 & 32 \\
\hline & Estudos em duplicata & 78 & 61 \\
\hline & Total de estudos identificados & 110 & 74 \\
\hline \multirow[t]{9}{*}{2} & Estudos excluídos & & \\
\hline & - idade inapropriada & 19 & 12 \\
\hline & - crianças com algum tipo de patologia & 16 & 4 \\
\hline & - suplementação com um único micronutriente & 13 & 5 \\
\hline & $\begin{array}{l}\text { - suplementação acompanhada de outra intervenção objeto } \\
\text { de avaliação ou suplementação exclusivamente durante a } \\
\text { gravidez }\end{array}$ & 13 & 3 \\
\hline & $\begin{array}{l}\text { - duração do seguimento inferior a oito semanas e/ou baixa } \\
\text { dose do suplemento }\end{array}$ & 1 & - \\
\hline & $\begin{array}{l}\text { - sem análise do efeito da suplementação no crescimento/ } \\
\text { estudo não experimental }\end{array}$ & 22 & - \\
\hline & $\begin{array}{l}\text { - sem enfoque na suplementação de micronutrientes/sem } \\
\text { análise do efeito da suplementação no crescimento/revisão } \\
\text { sistemática sem metanálise/outro estudo diferente de revisão }\end{array}$ & - & 47 \\
\hline & Total de estudos excluídos & 84 & 71 \\
\hline 3 & Estudos incluídos segundo busca nas bases de dados & 26 & 3 \\
\hline 4 & Estudos identificados e incluídos segundo listas de referências & 3 & 1 \\
\hline 5 & Total de estudos incluídos & 29 & 4 \\
\hline
\end{tabular}

crianças menores de 12 meses. Quanto aos efeitos sobre o crescimento ponderal, de um total de 10 estudos, 14,16-20,29,31,32,38 somente um 14 mostrou efeito positivo. Nenhum dos dez estudos que analisaram os efeitos da suplementação múltipla sobre o crescimento global,14,16-18,20,29-32,38 mostrou efeito positivo.

\section{II- Suplementação tripla ou dupla vs placebo}

De um total de 13 estudos,23-28,33,35-37,39-41 dois 24,39 demostraram o efeito positivo da suplementação tripla com zinco + ferro + vit. A no crescimento linear de crianças com déficit de estatura, um ${ }^{24}$ mostrou efeito positivo da suplementação com zinco + ferro em crianças com déficit de estatura e um 40 mostrou efeito positivo da suplementação com zinco + vit. A em crianças com deficiências de vit. A e de zinco. De um total de 12 estudos,23,24,26-28,33,35-
37,39-41 que analisou o efeito dessa intervenção sobre o crescimento ponderal, dois 35,40 demonstraram o efeito positivo da suplementação com zinco + vit. A em crianças com déficit de estatura 35 e em crianças com deficiências de vit. A e de zinco. ${ }^{40}$ Com relação aos 12 estudos, $23-28,33,36,37,39-41$ que avaliaram o efeito dessa modalidade de suplementação no crescimento global, um 40 mostrou o efeito positivo da combinação de zinco + vit. A em crianças com deficiências desses nutrientes.

\section{III- Suplementação com múltiplos micronu-} trientes vs suplementação tripla, dupla ou única

$\overline{\text { Entre }}$ os sete estudos que avaliaram o efeito da suplementação com múltiplos nutrientes sobre o crescimento linear, 15,21,22,29-32 um 15 mostrou o maior efeito da suplementação com múltiplos micronutri- 


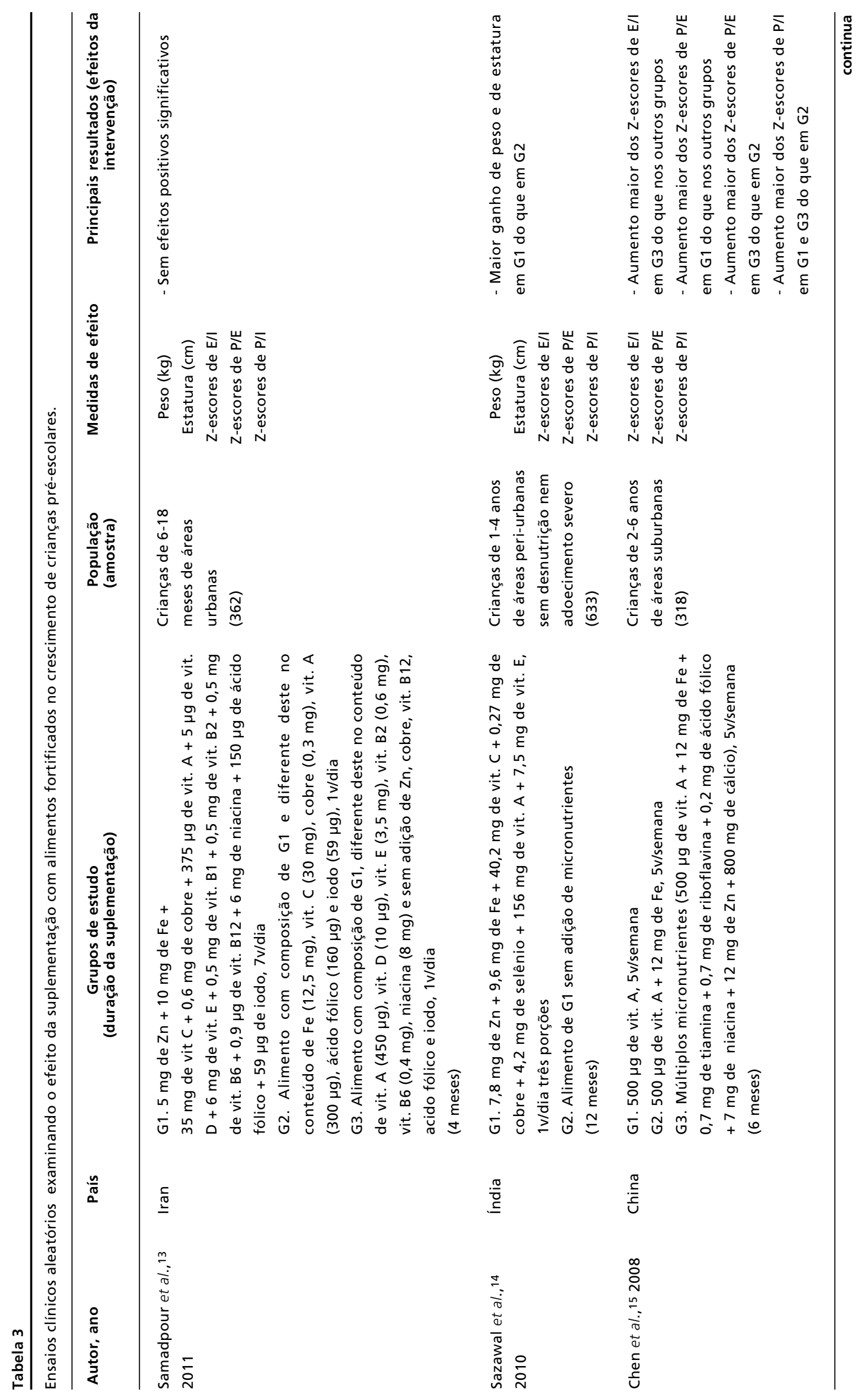




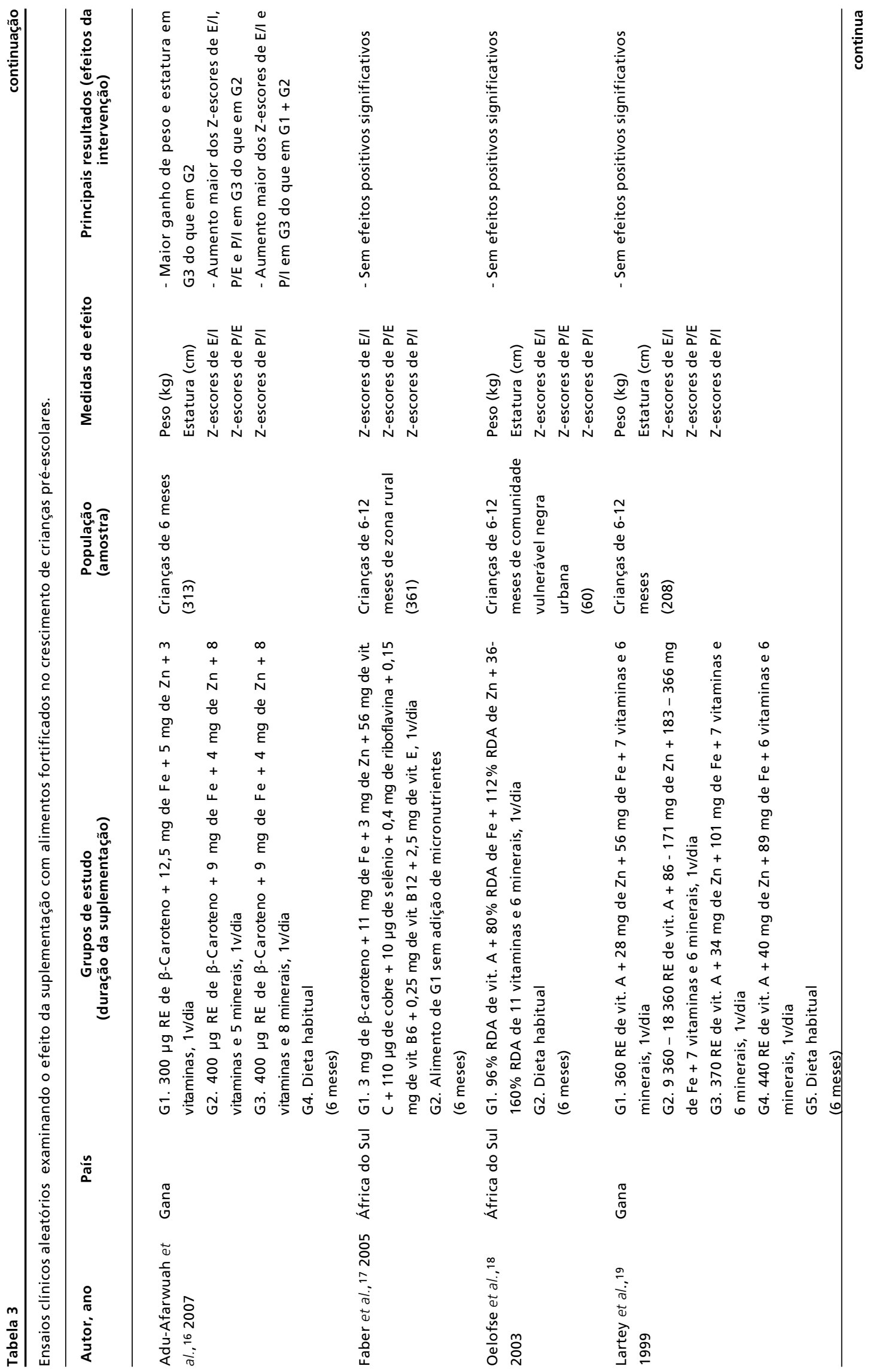




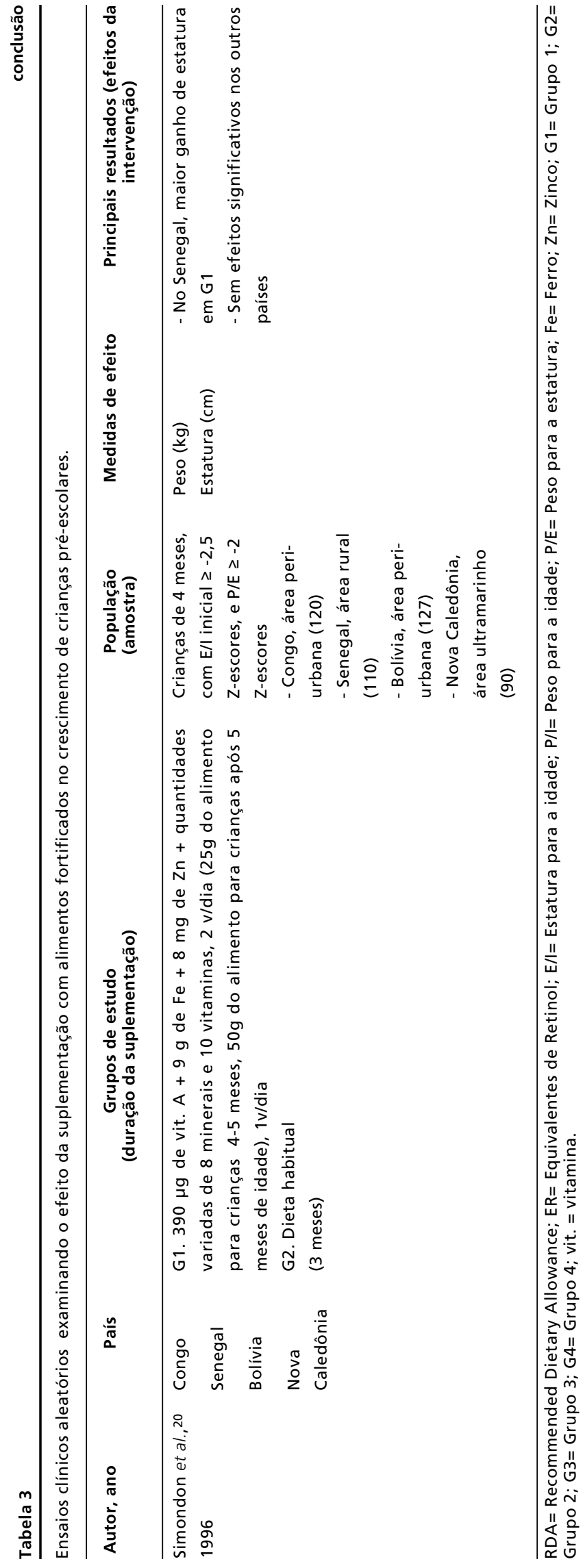




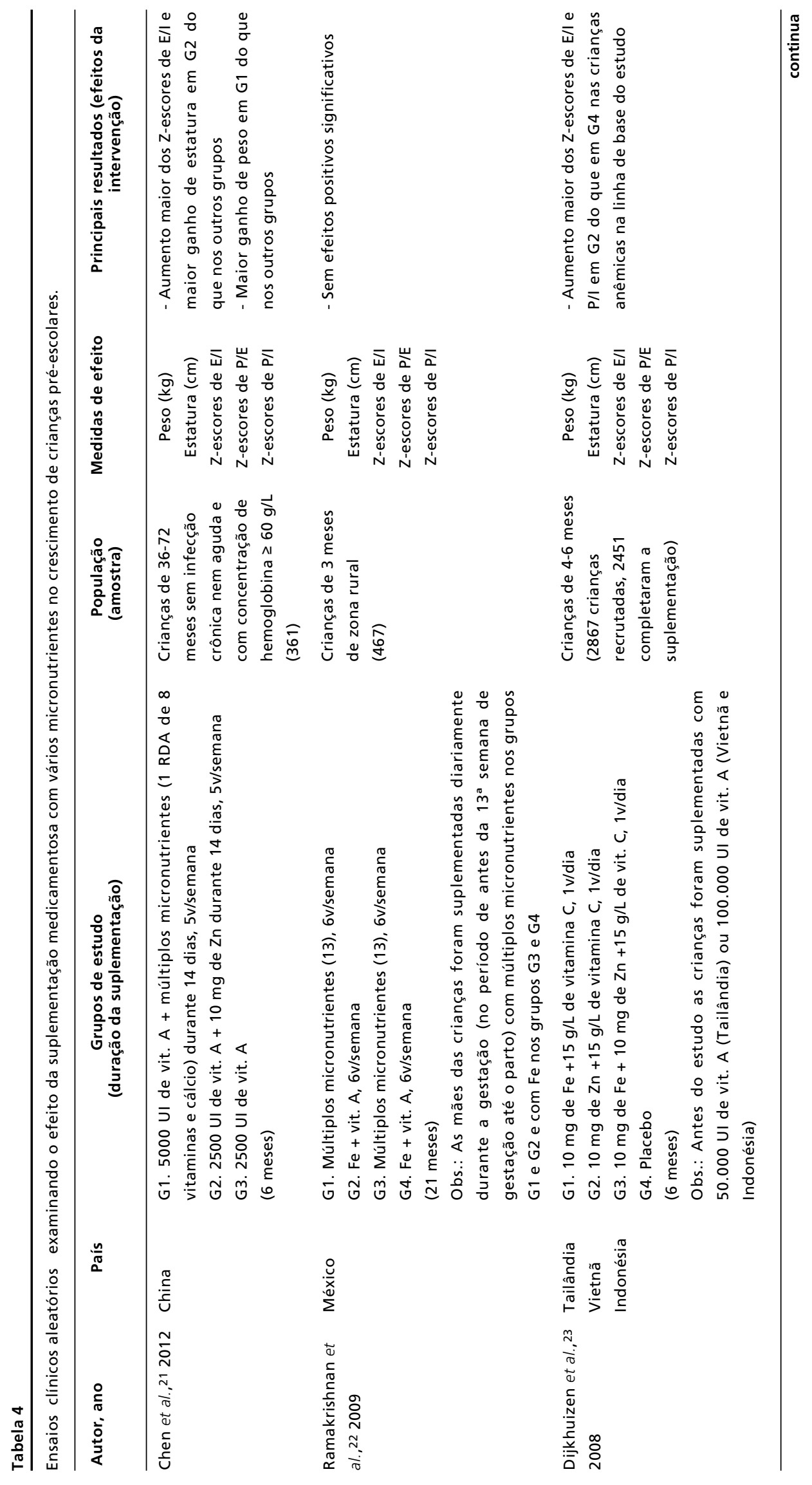




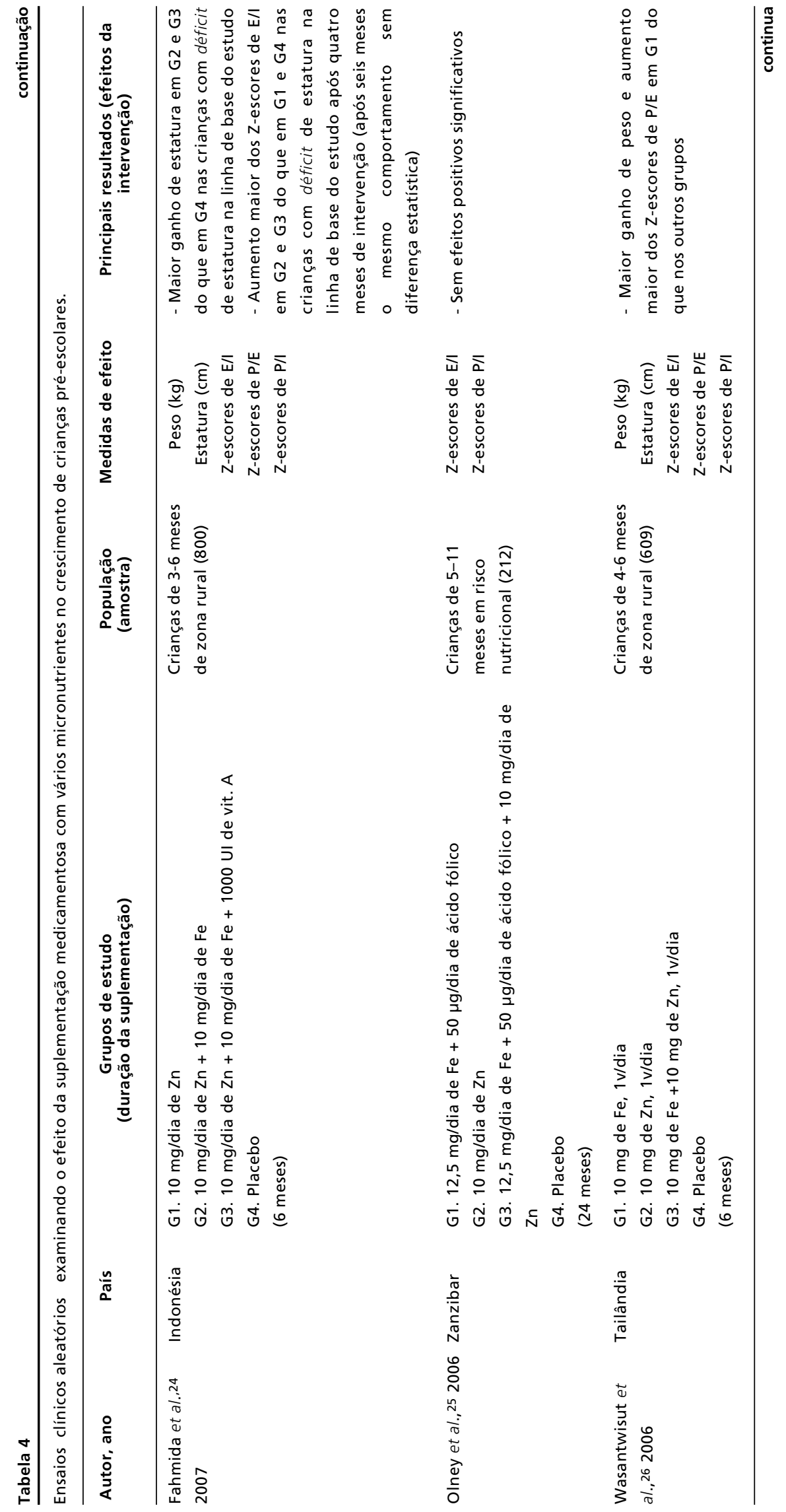




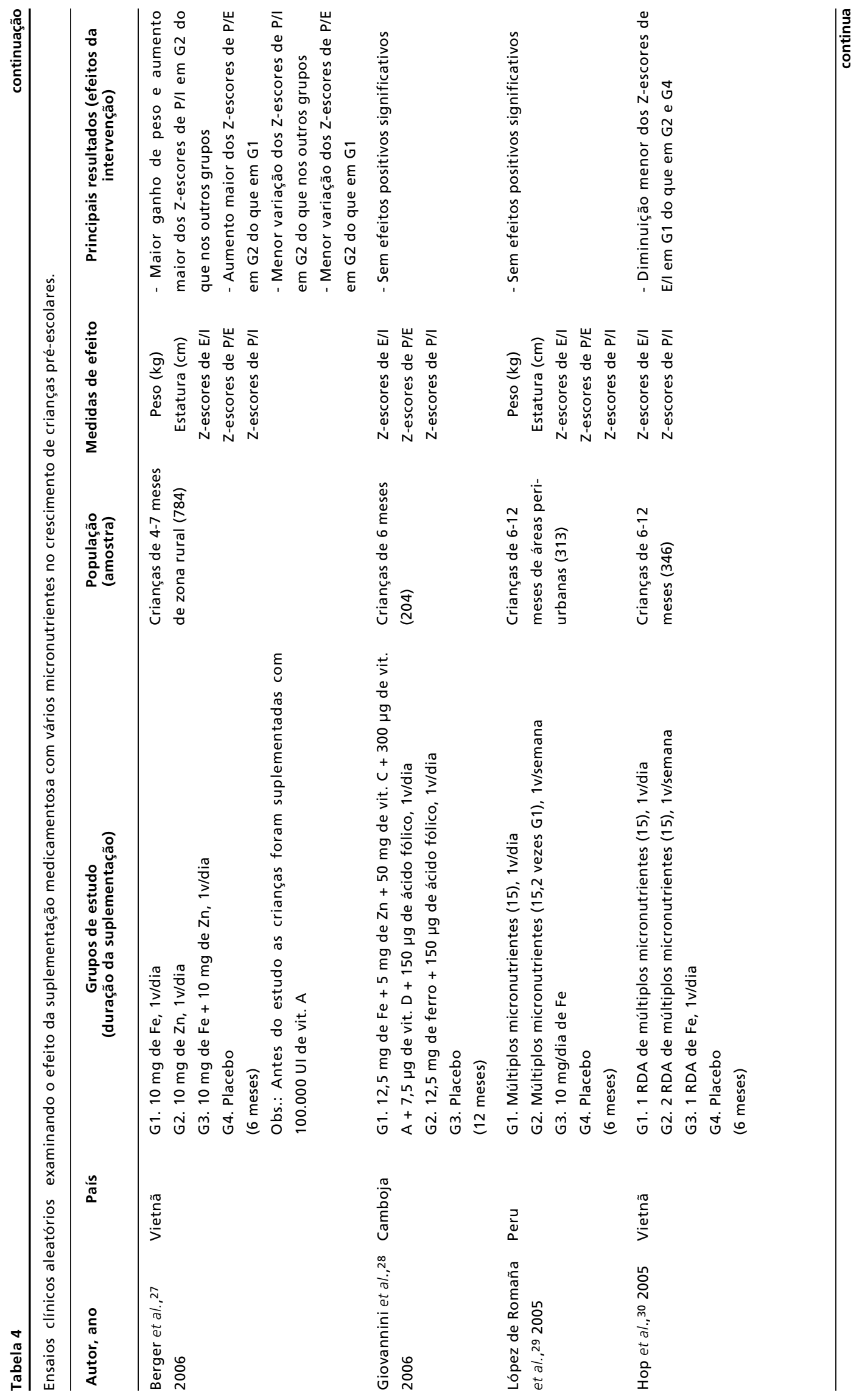




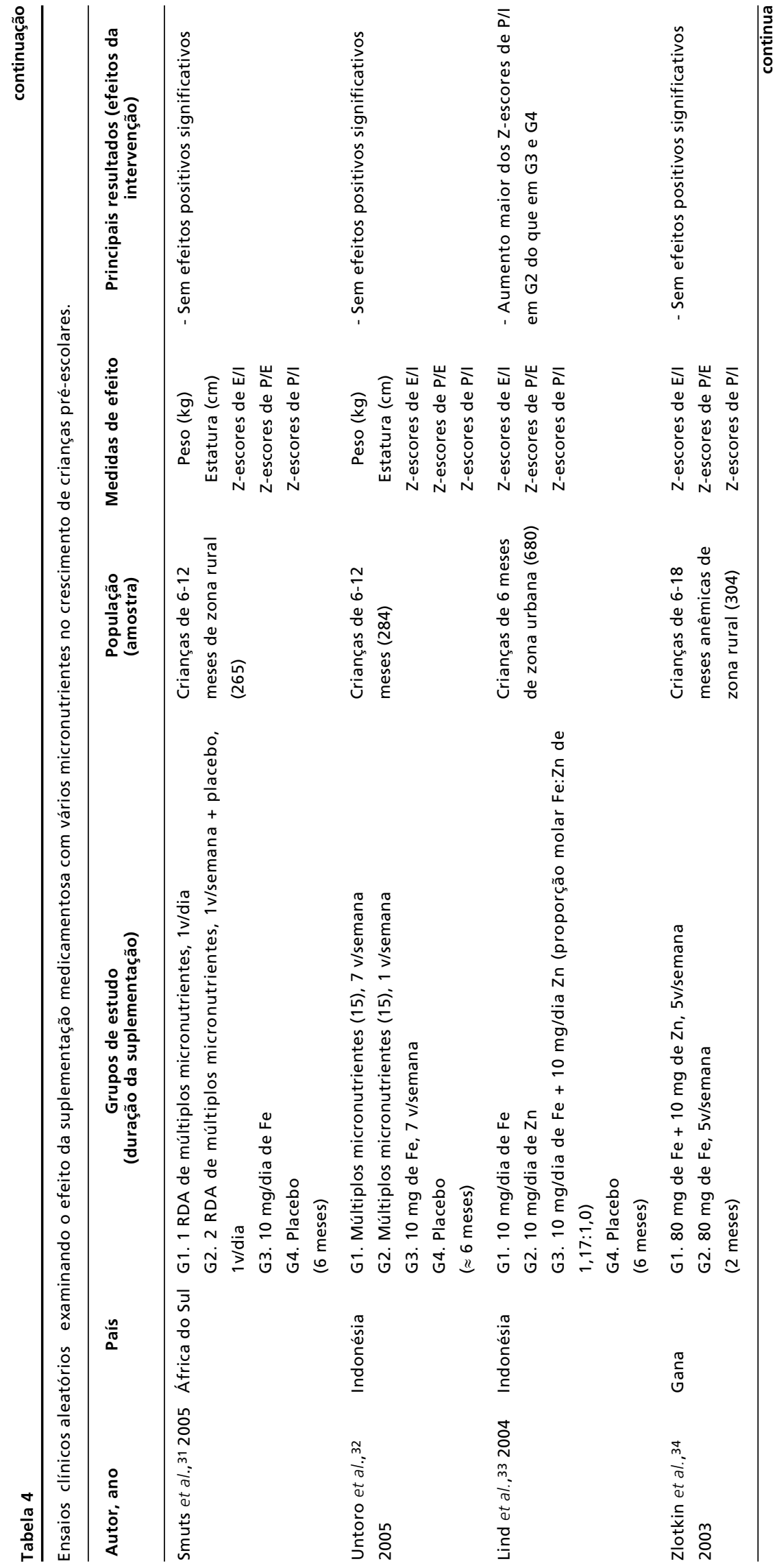




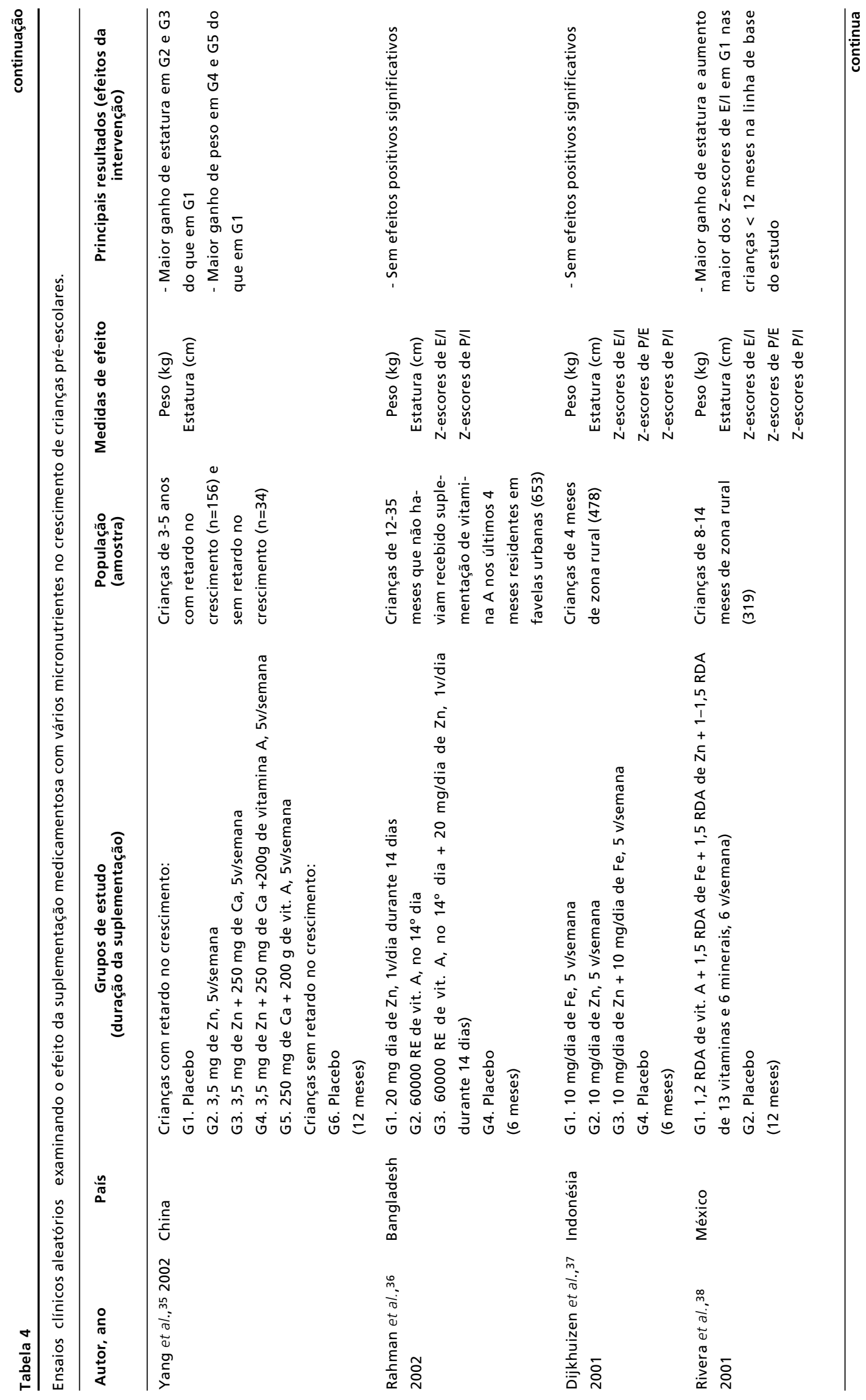




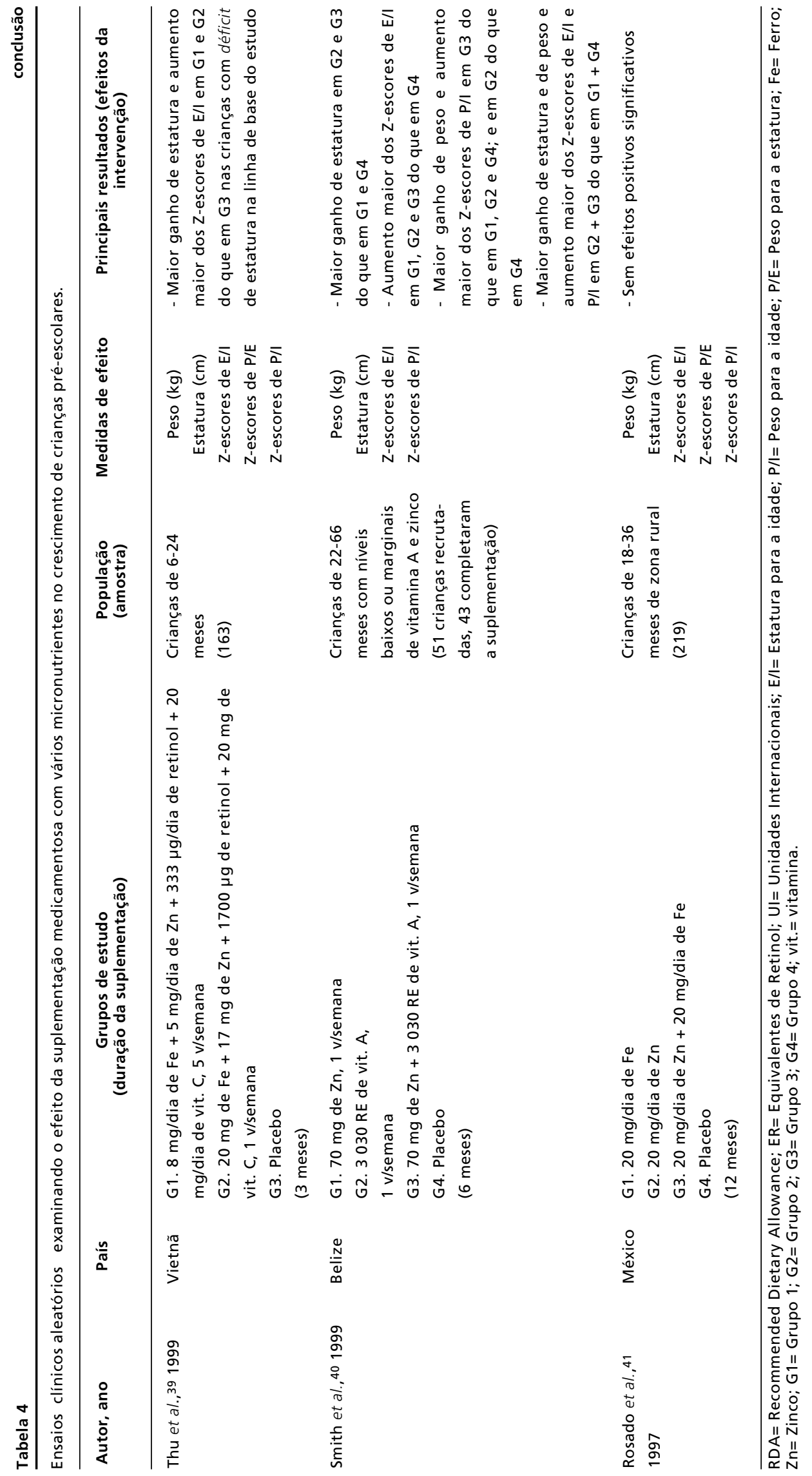


entes do que a suplementação com ferro + vit. A e com relação à suplementação com vit. $\mathrm{A}, \mathrm{e}$ um ${ }^{21}$ mostrou o maior efeito da suplementação com zinco + vit. A em relação à suplementação com múltiplos micronutrientes. Entre seis estudos, 15,21,22,29,31,32 que avaliaram o efeito dessa intervenção sobre o crescimento ponderal, um 15 verificou o maior efeito da suplementação com múltiplos micronutrientes quando comparada à combinação de ferro + vit. A e o maior efeito da suplementação com vit. A em relação à suplementação com múltiplos micronutrientes, e um ${ }^{21}$ mostrou maior efeito da suplementação com múltiplos micronutrientes do que com zinco + vit. A e do que com vit. A. De um total de sete estudos que analisaram o efeito da suplementação múltipla sobre o o crescimento global, 15,21,22,29-32 um 15 mostrou o maior efeito deste esquema em relação à suplementação com ferro + vit. A.

\section{IV- Suplementação tripla vs suplementação} dupla ou única

Um total de dois estudos avaliaram o impacto da suplementação tripla sobre o crescimento linear,24,28 dos quais um 24 mostrou maior o efeito da suplementação com zinco + ferro + vit. A do que com o esquema com zinco em crianças com déficit de estatura. Dos dois estudos identificados que testaram o impacto da suplementação tripla sobre o crescimento ponderal,24,28 nenhum mostrou a superioridade desse esquema em relação aos grupos de comparação. Do mesmo modo, nenhum dos dois estudos que avaliaram o impacto desta suplementação no crescimento global,24,28 demostraram o maior efeito dessa intervenção.

\section{V- Suplementação dupla vs suplementação}

\section{única}

$\overline{\text { Entre }}$ os 14 ensaios que avaliaram os efeitos da suplementação dupla em relação à suplementação única, 15,21,23-27,33-37,40,41 no crescimento linear, um ${ }^{24}$ mostrou maior efeito da suplementação com zinco + ferro quando comparado à suplementação com zinco em crianças com déficit de estatura, um 40 mostrou maior efeito da suplementação com zinco + vit. A do que com zinco, em crianças com deficiências de vit. A e de zinco e um ${ }^{21}$ mostrou maior efeito da suplementação com zinco + vit. A do que com a vit. A. Quanto aos 14 estudos que avaliaram os efeitos deste esquema sobre o crescimento ponderal, 15,21,23-27,33-37,40,41 um 40 verificou o maior efeito da suplementação com zinco + vit. A quando comparada a suplementação com zinco e à suplementação com vit. A, em crianças com deficiências de zinco e de vit. A, um 27 demonstrou o maior efeito da suplementação com zinco do que a combinação de zinco + ferro, um ${ }^{26}$ mostrou maior efeito da suplementação com ferro do que com ferro + zinco, e um ${ }^{15}$ mostrou maior efeito da suplementação com vit. A do que com vit. A + ferro. Em relação aos 12 estudos que testaram o efeito da suplementação dupla sobre o crescimento global,15,23$27,33,34,36,37,40,41$ dois 27,33 mostraram o menor efeito da suplementação com zinco + ferro do que com zinco, um ${ }^{40}$ mostrou maior efeito da suplementação com zinco + vit. A do que a suplementação com zinco ou a suplementação com vit. A, em crianças com deficiências de vit. A e de zinco, e um ${ }^{15}$ mostrou maior efeito da suplementação com vit. A do que com a combinação de vit. A + ferro.

\section{Estudos de revisão sistemática com metanálise

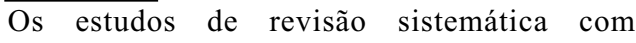
metanálise incluídos42-45 estão caracterizados na Tabela 5. Todos os quatro estudos investigaram o impacto da suplementação com múltiplos micronutrientes $v s$ placebo sobre o crescimento de crianças. Três estudos 43-45 evidenciaram o impacto positivo da suplementação múltipla no crescimento linear, enquanto que dois apresentaram resultado similar sobre o crescimento ponderal.44,45 Allen et al.44 constataram o maior efeito da suplementação com múltiplos micronutrientes $v s$ suplementação dupla ou única no crescimento linear e ponderal, exceto o efeito no crescimento linear de múltiplos micronutrientes $v s$ ferro + ácido fólico. Ramakrishnan et al. 43 analisando dez estudos que analisaram o efeito da suplementação dupla, de ferro + zinco ou vit. A + zinco, $v s$ placebo, não indicaram qualquer efeito positivo sobre o crescimento linear ou ponderal. Em nenhum dos estudos os impactos indicados no crescimento linear 44,45 e no crescimento ponderal 45 estiveram condicionados ao estado nutricional das crianças.

\section{Discussão}

$\mathrm{Na}$ maioria dos países em desenvolvimento e em alguns grupos populacionais de países desenvolvidos, a alimentação habitual é insuficiente para suprir $100 \%$ dos micronutrientes requeridos para as crianças, principalmente os minerais ferro, zinco e cálcio, e em menor proporção algumas vitaminas, incluindo a vit. A. ${ }^{46}$ Este dado alerta para a necessidade de, em algumas situações, se considerar a administração de suplementos nutricionais para aperfeiçoar o potencial genético de crescimento físico e prevenir o surgimento de doenças infec- 


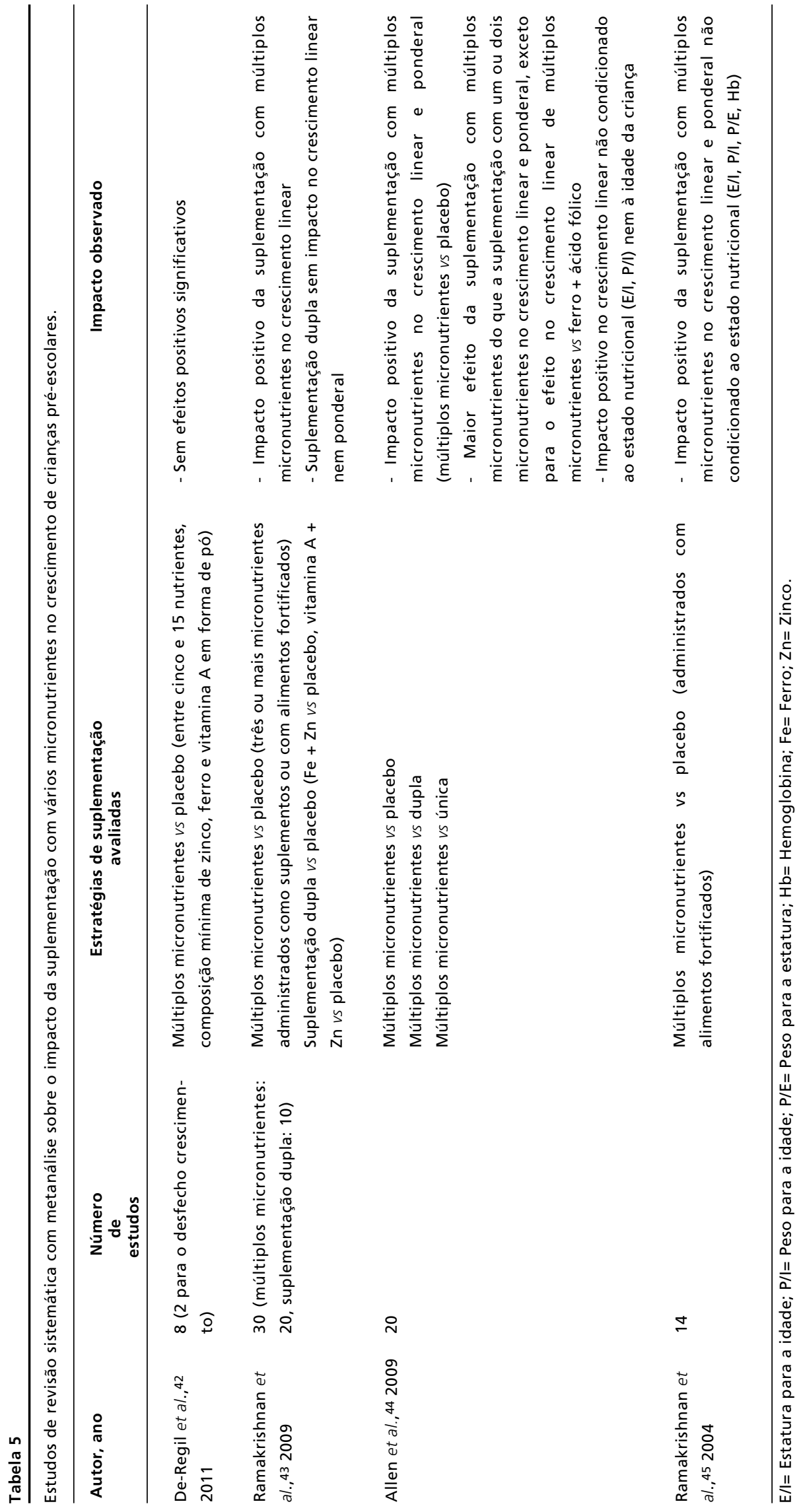


ciosas, além de ações de estímulo ao aleitamento materno, melhoria das condições sanitárias e da qualidade da dieta alimentar. 46,47

Entretanto, estudos de revisão sistemática têm fornecido evidências da ausência de efeito benéfico da suplementação isolada com ferro 48 ou com vit. $\mathrm{A}^{45}$ sobre o crescimento. Com relação ao zinco, as resultados dos estudos têm sido contraditórios, demonstrando tanto seu efeito positivo, 49 quanto a ausência de impacto. ${ }^{43}$ Como a condição nutricional pode influenciar a resposta a uma determinada intervenção, 50 além de fatores como a variabilidade das intervenções (inclusive o tempo de intervenção), seleção de grupos controle, faixa etária e características do local de estudo, a melhoria do estado nutricional da população de crianças, observada ao longo dos anos, pode ser uma possível explicação para tais resultados. ${ }^{43}$ Assim, estratégias voltadas à melhoria e à prevenção dos problemas relacionados ao crescimento derivadas de políticas públicas seriam importantes, considerando-se que os benefícios da suplementação com micronutrientes isolados são pequenos. Nesse sentido, a suplementação com micronutrientes ainda é destacada por constituir uma medida custo-efetiva, 43,46 tornando-se necessária a realização de mais estudos que investiguem o papel da suplementação com micronutrientes sobre o crescimento na população de crianças.

Nesta revisão, os resultados das análises dos ensaios clínicos controlados evidenciam que a administração de suplementos nutricionais contendo combinações de zinco, ferro e vit. A pode aumentar o potencial de crescimento das crianças. Apesar das análises mostrarem que esses efeitos são mais evidentes quando a suplementação é condicionada à vulnerabilidade biológica (menor idade) e/ou nutricional (deficiências de micronutrientes, déficit de estatura), não houve sinergismo desses achados com as conclusões obtidas nos estudos de revisão sistemática com metanálise. Rivera Dommarco et al.,4 através da revisão de cinco estudos que avaliaram múltiplos micronutrientes $v s$ placebo concluíram, também, sobre o impacto positivo da suplementação no crescimento linear condicionado a crianças menores de 12 meses e a crianças com déficit de estatura. Para outras estratégias de intervenção, resultados similares foram apontados pelos pesquisadores. 51

Com base nos dados dos ensaios clínicos controlados, constatou-se que a mais forte evidência do efeito positivo da suplementação múltipla sobre o crescimento proveio das comparações da suplementação com múltiplos micronutrientes $v s$ placebo ou dieta habitual, principalmente em relação ao cresci- mento linear de crianças menores de 12 meses. Ao contrário, a comparação dos efeitos da suplementação tripla ou dupla vs placebo não mostrou o maior efeito dessa intervenção. Esses resultados convergem com as evidências fornecidas pelas revisões sistemáticas selecionadas neste estudo, em relação ao efeito da suplementação com múltiplos micronutrientes $v s$ placebo, bem como em relação à à ausência de evidências do impacto positivo da suplementação dupla vs placebo. Esses resultados são importantes, pois além de fortalecerem as evidências sobre o efeito benéfico da estratégia de suplementação múltipla com micronutrientes indicam importantes semelhanças metodológicas na condução das pesquisas.

A análise dos ensaios que analisaram os efeitos da suplementação com múltiplos micronutrientes vs suplementação tripla, dupla ou única chamam a atenção para a necessidade de se conduzir mais estudos comparando o efeito dessas estratégias. Entretanto, o maior efeito desta suplementação em relação à suplementação dupla ou única (exceto para o ferro) ficou claramente evidenciada no estudo conduzido por Allen et al.44

Em relação à suplementação dupla $v s$ suplementação única, os resultados sugerem que o efeito da suplementação com um único micronutriente não parece ser significativo nas crianças com coexistência de deficiências de micronutrientes ou com déficit de estatura. Além disso, mostra que o efeito da suplementação com zinco e ferro ou com ferro e vit. A sobre o crescimento não parece ser maior entre as crianças eutróficas, ou seja, a suplementação com um único micronutriente parece ter maior efeito, enquanto que a suplementação com zinco e vit. A possui efeito positivo. Essas conclusões necessitam ser confirmadas ou refutadas em futuros estudos de revisão sistemática com metanálise. É importante destacar que a interpretação desses resultados levou em consideração as interações existentes entre os micronutrientes e o efeito negativo de alguns deles na absorção de outros. O ferro e o zinco apresentam mecanismos de absorção e transporte similares, podendo o ferro inibir a absorção ou biodisponibilidade de zinco e, portanto, o seu efeito no crescimento linear quando suplementados de forma simultânea. ${ }^{49}$ A vit. A, por sua vez, apresenta inte-ração positiva na absorção e função do zinco. ${ }^{21}$ Futuros estudos necessitam ser delineados de forma a considerar tais fatores, sobretudo em relação à combinação com ferro e vit. A.

Cabe ressaltar que as evidências dos efeitos benéficos da suplementação com micronutrientes se referem não apenas ao crescimento, mais igualmente 
no desenvolvimento mental e motor, 52 além da proteção contra doenças infecciosas. ${ }^{47}$ Além disso, deve-se considerar as repercussões benéficas de longo prazo da suplementação múltipla com micronutrientes, uma vez que a prevenção do déficit de estatura no início da vida evita complicações futuras como o acúmulo de gordura corporal, obesidade e doenças crônicas associadas. 53

Adicionalmente, o efeito da idade, especialmente nos primeiros dois anos de vida, necessita ser um fator a ser considerado nas análises, uma vez que constitui um fator que influencia o crescimento independentemente do efeito da suplementação. Nessa fase da vida, existe maior vulnerabilidade a doenças infecciosas e desnutrição, maior chance de se obter efeitos positivos da suplementação, assim como maior chance de reversão de déficits de estatura e de danos decorrentes das deficiências de micronutrientes. $30,50,51,54$

No contexto da saúde pública cabe reforçar que as intervenções focadas na alimentação (fortificação, diversificação alimentar, educação alimentar e nutricional) apresentam vantagens relacionadas à adição de nutrientes na forma em que é consumido, o que facilita sua integração à dieta habitual e oferece a possibilidade de ser fonte adicional de energia e proteína de alta qualidade com efeitos diretos no sistema hormonal ligado ao crescimento. 4 Nesse sentido, esta estratégia de prevenção pode se apresentar mais vantajosa do que a suplementação com micronutrientes, em termos de custo-efetividade, 55 reconhecendo, ainda, a importância de sistemas alimentares sustentáveis para assegurar a segurança alimentar. 56 No entanto, cabe ressaltar que a opção por essa estratégia não reduz o importante papel da suplementação, sobretudo em determinados grupos, como as crianças com deficiências, nas quais a fortificação universal não oferece o aporte de micronutrientes adequados às suas necessidades desse grupo populacional. 55

A aplicação das evidências científicas na definição de políticas e programas (governamentais e não governamentais) de alimentação e nutrição direcionados às crianças pré-escolares é importante para aumentar a sua efetividade. Esses programas devem englobar ações de promoção do aleitamento materno exclusivo até os seis meses de idade, das boas práticas de alimentação complementar, a suplementação alimentar, além de ações voltadas às melhorias das condições de abastecimento de água, saneamento e higiene e a suplementação com micronutrientes, para a prevenção de doenças infecciosas e de problemas no crescimento linear. 47,51 As experiências de países, como o México e o Brasil, podem servir de exemplo de ações direcionadas à prevenção de deficiências de micronutrientes para outros países subdesenvolvidos ou em desenvolvimento. No México, o programa nacional de fortificação com zinco e outros micronutrientes realiza adição desses nutrientes às farinhas de trigo e milho, que são usadas na preparação do pão e tortilhas, os dois principais alimentos no país. 57 No Brasil, destacam-se o Programa Nacional de Suplementação de Ferro, que preconiza a suplementação com ferro às gestantes e crianças até 18 meses de vida, o Programa Nacional de Suplementação de Vitamina A, que prevê a suplementação com essa vitamina a crianças com até cinco anos e puérperas residentes em regiões de vulnerabilidade,e o programa de fortificação das farinhas com ferro e ácido fólico. 58,59 Entretanto, a recomendação da suplementação com múltiplos micronutrientes ainda é limitada. ${ }^{44}$ Desse modo, os resultados do presente trabalho tornam-se importantes no processo de tomada de decisão, na medida em que buscam fornecer evidências dos efeitos da suplementação com múltiplos micronutrientes.

No âmbito da definição das políticas, apesar de reconhecermos as limitações do índice peso/idade na identificação da natureza do agravo nutricional, considera-se seu uso importante por constituir uma medida sensível dos problemas nutricionais nos primeiros anos da vida, que tem sido utilizada mundialmente no acompanhamento individual do crescimento de crianças e na avaliação do impacto de intervenções nutricionais. 59

Conclui-se que apesar de existirem fortes evidências dos efeitos benéficos do zinco, ferro e vit. A, bem como das estratégias de suplementação com esses micronutrientes na saúde e bem estar das crianças, mais estudos são necessários para uma melhor discriminação dos efeitos desses micronutrientes no crescimento. Conclui-se também que existem evidências sobre os efeitos positivos da suplementação com múltiplos micronutrientes sobre o crescimento linear, particularmente quando comparada à suplementação dupla ou única. Quanto à suplementação dupla, os resultados mostram que a combinação de zinco e vit. A constitui a melhor opção devido aos mecanismos absortivos positivos. As questões mais importantes para futuras pesquisas se referem ao efeito da suplementação tripla em relação às outras opções (placebo, única, dupla, múltipla) e à possível influencia da condição nutricional e/ou idade das crianças na análise dos efeitos da suplementação. O esclarecimento dessas questões são relevantes para orientar estratégias de prevenção tecnicamente factíveis e custo-efetivas. 


\section{Referências}

1. Macêdo EMC. Efeitos da deficiência de cobre, zinco e magnésio sobre o sistema imune de crianças com desnutrição grave. Rev Paul Pediatr. 2010; 28 (3): 329-36.

2. Orlonski S, Dellagrana RA, Rech CR, Araújo ED. Estado nutricional e fatores associados ao déficit de estatura em crianças atendidas por uma unidade de ensino básico de tempo integral. Rev Bras Crescimento Desenvolv Hum. 2009; 19 (1): 54-62.

3. Bueno AL, Czepielewski MA. Micronutrientes envolvidos no crescimento. Rev HCPA. 2007; 27 (3): 47-56

4. Rivera Dommarco J, Hotz C, Gonzalez de Cossío T, Neufeld L, Garcia Guerra A. The effect of micronutrient deficiencies on child growth: a review of results from community-based supplementation trials. J Nutr. 2003; 133 (11 Suppl. 2): 4010-20.

5. Sena KCM, Pedrosa LFC. Efeitos da suplementação com zinco sobre o crescimento, sistema imunológico e diabetes. Rev Nutr. 2005; 18 (2): 251-9.

6. Silva LSV, Thiapó AP, Souza GG, Sauders C, Ramalho A. Micronutrientes na gestação e lactação. Rev Bras Sáude Matern Infant. 2007; 7 (3): 237-44.

7. Bhan MK, Sommerfelt H, Strand T. Micronutrient deficiency in children. Br J Nutr. 2001; 85 (2): 199-03.

8. Dijkhuizen MA, Wieringa FT, West CE. Concurrent micronutrient deficiencies in lactating mothers and their infants in Indonesia. Am J Cli Nutr. 2001; 73: 786-91.

9. Gibson RS, Hotz C. Nutritional causes of linear growth faltering in infants during the complementary feeding period. In: Martorell R, Haschke F, editors. Nutrition and Growth. Nestle Nutrition Workshop Series Pediatric Program. Philadelphia: Lippincott, Williams \& Wilkins; 2001. p.159-92.

10. Hadi H, Stoltzfus RJ, Dibley MJ, Moulton LH, West KP Jr, Kjolhede CL, Sadjimin T. Vitamin A supplementation selectively improves the linear growth of Indonesian preschool children: results from a randomized controlled trial. Am J Clin Nutr. 2000; 71 (2): 507-13.

11. Lindsay H. Allen LH, Peerson JM, Olney DK. Provision of multiple rather than two or fewer micronutrients more effectively improves growth and other outcomes in micronutrient-deficient children and adults. J Nutr. 2009; 139: 1022-30

12. Branca F, Ferrari M. Impact of micronutrient deficiencies on growth: the Stunting Syndrome. Ann Nutr Metab. 2002; 46 (Suppl. 1): 8-17.

13. Samadpour K, Long KZ, Hayatbakhsh R, Marks GC. Randomised comparison of the effects of Sprinkles and Foodlets with the currently recommended supplement (Drops) on micronutrient status and growth in Iranian children. Eur J Clin Nut. 2011; 65 (12): 1287-94.

14. Sazawal S, Dhingra U, Dhingra P, Hiremath G, Sarkar A, Dutta A, Menon VP, Black RE. Micronutrient fortified milk improves iron status, anemia and growth among children 14 years: a double masked, randomized, controlled trial. PLoS One. 2010; 5 (8): e12167.

15. Chen K, Li TY, Chen L, Qu P, Liu YX. Effects of vitamin A, vitamin A plus iron and multiple micronutrient-fortified seasoning powder on preschool children in a suburb of
Chongqing, China. J Nutr Sci Vitaminol. 2008; 54 (6): 440-

16. Adu-Afarwuah S, Lartey A, Brown KH, Zlotkin S, Briend A, Dewey KG. Randomized comparison of 3 types of micronutrient supplements for home fortification of complementary foods in Ghana: effects on growth and motor development. Am J Clin Nutr. 2007; 86: 412-20.

17. Faber M, Kvalsvig JD, Lombard CJ, Benadé AJ. Effect of a fortified maize-meal porridge on anemia, micronutrient status, and motor development of infants. Am J Clin Nutr. 2005; 82 (5): 1032-9.

18. Oelofse A, Van Raaij JM, Benadé AJ, Dhansay MA, Tolboom JJ, Hautvast JG. The effect of a micronutrientfortified complementary food on micronutrient status, growth and development of 6- to 12-month-old disadvantaged urban South African infants. Int J Food Sci Nutr. 2003; 54 (5): 399-407.

19. Lartey A, Manu A, Brown KH, Peerson JM, Dewey KG. A randomized, community-based trial of the effects of improved, centrally processed complementary foods on growth and micronutrient status of Ghanaian infants from 6 to 12 mo of age. Am J Clin Nutr. 1999; 70: 391-404.

20. Simondon KB, Gartner A, Berger J, Cornu A, Massamba A, San Miguel JL, et al. Effect of early, short-term supplementation on weight and linear growth of 4-7-mo-old infants in developing countries: a four-country randomized trial. Am J Clin Nutr. 1996; 64 (4): 537-45.

21. Chen L, Liu YF, Gong M, Jiang W, Fan Z, Qu P, Chen J, Liu YX, Li TY. Effects of vitamin A, vitamin A plus zinc, and multiple micronutrients on anemia in preschool children in Chongqing, China. Asia Pac J Clin Nutr. 2012; 21 (1): 3-11.

22. Ramakrishnan U, Neufeld LM, Flores R, Rivera J, Martorell R. Multiple micronutrient supplementation during early childhood increases child size at 2 y of age only among high compliers. Am J Clin Nutr. 2009; 89 (4): 1125-31.

23. Dijkhuizen MA, Winichagoon P, Wieringa FT, Wasantwisut E, Utomo B, Ninh NX, Hidayat A, Berger J. Zinc supplementation improved length growth only in anemic infants in a multi-country trial of iron and zinc supplementation in South-East Asia. J Nutr. 2008; 138 (10): 1969-75.

24. Fahmida U, Rumawas J, Utomo B, Patmonodewo S, Schultink W. Zinc-iron, but not zinc-alone supplementation, increased linear growth of stunted infants with low haemoglobin. Asia Pac J Clin Nutr. 2007; 16 (2): 301-9.

25. Olney DK, Pollitt E, Kariger PK, Khalfan SS, Ali NS, Tielsch JM, Sazawal S, Black R, Allen LH, Stoltzfus RJ. Combined iron and folic acid supplementation with or without zinc reduces time to walking unassisted among Zanzibari infants 5- to 11-mo old. J Nutr. 2006; 136 (9): 2427-34.

26. Wasantwisut E, Winichagoon $\mathrm{P}$, Chitchumroonchokchai $\mathrm{C}$, Yamborisut U, Boonpraderm A, Pongcharoen T, Sranacharoenpong K, Russameesopaphorn W. Iron and zinc supplementation improved iron and zinc status, but not physical growth, of apparently healthy, breast-fed infants in rural communities of northeast Thailand. J Nutr. 2006; 136 (9): 2405-11 
27. Berger J, Ninh NX, Khan NC, Nhien NV, Lien DK, Trung NQ, Khoi HH. Efficacy of combined iron and zinc supplementation on micronutrient status and growth in Vietnamese infants. Eur J Clin Nutr. 2006; 60 (4): 443-54.

28. Giovannini M, Sala D, Usuelli M, Livio L, Francescato G, Braga M, Radaelli G, Riva E. Double-blind, placebocontrolled trial comparing effects of supplementation with two different combinations of micronutrients delivered as sprinkles on growth, anemia, and iron deficiency in cambodian infants. J Pediatr Gastroenterol Nutr. 2006; 42 (3): 306-12.

29. López de Romaña G, Cusirramos S, López de Romaña D, Gross R. Efficacy of multiple micronutrient supplementation for improving anemia, micronutrient status, growth, and morbidity of Peruvian infants. J Nutr. 2005; 135 (Suppl. 3): 646S-52.

30. Hop le T, Berger J. Multiple micronutrient supplementation improves anemia, micronutrient nutrient status, and growth of Vietnamese infants: double-blind, randomized, placebocontrolled trial. J Nutr. 2005; 135 (Suppl. 3): 660S-5.

31. Smuts CM, Dhnasay MA, Faber M, van Stuijvenberg ME, Swanevelder S, Gross R, Benadé AJ. Efficacy of multiple micronutrient supplementation for improving anemia, micronutrient status, and growth in South African infants. J Nutr. 2005; 135 (Suppl. 3): 653S-9.

32. Untoro J, Karyadi E, Wibowo L, Erhardt MW, Gross R. Multiple micronutrient supplements improve micronutrient status and anemia but not growth and morbidity of Indonesian infants: a randomized, double-blind, placebocontrolled trial. J Nutr. 2005; 135 (Suppl. 3): 639S-45.

33. Lind T, Lönnerdal B, Stenlund H, Gamayanti IL, Ismail D, Seswandhana R, Persson LA. A community-based randomized controlled trial of iron and zinc supplementation in Indonesian infants: effects on growth and development. Am J Clin Nutr. 2004; 80: 729-36.

34. Zlotkin S, Arthur P, Schauer C, Antwi KY, Yeung G, Piekarz A. Home-fortification with iron and zinc sprinkles or iron sprinkles alone successfully treats anemia in infants and young children. J Nutr. 2003; 133 (4): 1075-80.

35. Yang YX, Han JH, Shao XP, He M, Bian LH, Wang Z, Wang GD, Men JH. Effect of micronutrient supplementation on the growth of preschool children in China. Biomed Environ Sci 2002; 15 (3): 196-02.

36. Rahman MM, Tofail F, Wahed MA, Fuchs GJ, Baqui AH, Alvarez JO. Short-term supplementation with zinc and vitamin A has no significant effect on the growth of undernourished Bangladeshi children. Am J Clin Nutr. 2002; 75 (1): 87-91.

37. Dijkhuizen MA, Wieringa FT, West CE, Martuti S, Muhilal S. Effects of Iron and Zinc Supplementation in Indonesian Infants on Micronutrient Status and Growth. J Nutr. 2001; 131: $2860-5$

38. Rivera JA, González-Cossío T, Flores M, Romero M, Rivera M, Téllez-Rojo MM, Rosado JL, Brown KH Multiple micronutrient supplementation increases the growth of Mexican infants. Am J Clin Nutr. 2001; 74: $657-$ 63.

39. Thu B, Schultink W, Dillon D, Gross R, Leswara ND, Khoi $\mathrm{HH}$. Effect of daily and weekly micronutrient supplementation on micronutrient deficiencies and growth in young
Vietnamese children. Am J Clin Nutr. 1999; 69: 80-6.

40. Smith CJ, Makdani D, Hegar A, Rao D, Douglass LW. Vitamin A and Zinc Supplementation of Preschool Children. J American Coll Nut. 1999; 18(3): 213-22.

41. Rosado JL, Lopez P, Muñoz E, Martinez H, Allen LH. Zinc supplementation reduced morbidity, but neither zinc nor iron supplementation affected growth or body composition of Mexican preschoolers. Am J Clin Nutr. 1997; 65: 13-9.

42. De-Regil LM, Suchdev PS, Vist GE, Walleser S, PeñaRosas JP. Home fortification of foods with multiple micronutrient powders for health and nutrition in children under two years of age. Cochrane Database Syst Rev. 2011; Issue 9: CD008959.

43. Ramakrishnan U, Nguyen P, Martorell R. Effects of micronutrients on growth of children under 5 y of age: meta-analyses of single and multiple nutrient interventions. Am J Clin Nutr. 2009; 89: 191-03.

44. Allen LH, Peerson JM, Olney DK. Provision of multiple rather than two or fewer micronutrients more effectively improves growth and other outcomes in micronutrient-deficient children and adults. J Nutr. 2009; 139: 1022-30.

45. Ramakrishnan U, Aburto N, McCabe G, Martorell R. Multimicronutrient interventions but not vitamin A or iron interventions alone improve child growth: results of 3 metaanalyses. J Nutr. 2004; 134: 2592-602.

46. Allen LH, Gillespie SR. What works? A review of the efficacy and effectiveness of nutrition interventions. Manila, Philippines: ADB; 2001.

47. Imdad A, Sadig K, Bhutta ZA. Evidence-based prevention of childhood malnutrition. Curr Opin Clin Nutr Metab Care. 2011; 14 (3): 276-85.

48. Sachdev HPS, Gera T, Nestel P. Effect of iron supplementation on physical growth in children: systematic review of randomised controlled trials. Public Health Nutr. 2006; 9 (7): 904-20

49. Imdad A, Bhutta ZA. Effect of preventive zinc supplementation on linear growth in children under 5 years of age in developing countries: a meta-analysis of studies for input to the lives saved tool. BMC Public Health. 2011; 11 (Suppl. 3): S22.

50. Queiroz D, Paiva AA, Gama JSFA, Lima ZN, Figueroa Pedraza D. Índices antropométricos e retinolemia em crianças menores de cinco anos do estado da Paraíba. Rev Nut. 2013; 26 (5): 563-70.

51. Valle NJ, Santos IS, Gigante DP. Intervenções nutricionais e crescimento infantil em crianças de até dois anos de idade: uma revisão sistemática. Cad Saúde Pública. 2004; 20 (6): 1458-67.

52. Szajewska H. The role of meta-analysis in the evaluation of the effects of early nutrition on mental and motor development in children. Am J Clin Nutr. 2011; 94 (Supp1. 6): 1889S-95.

53. Santos CDL. Clemente APG, Martins PA, Sawaya AL. Influência do déficit de estatura nos desvios nutricionais em adolescentes e pré-adolescentes. Rev Nutr. 2009; 22 (2): $187-94$

54. Victora C. Los mil días de oportunidad para intervenciones nutricionales. De la concepción a los dos años de vida. Arch Argent Pediatr. 2012; 110 (4): 311-7 
55. Valencia-Mendoza A, Danese-dlSantos LG, Sosa-Rubí SG Aracena-Genao B. Costo-efectividad de prácticas en salud pública: revisión bibliográfica de las intervenciones de la Iniciativa Mesoamericana de Salud. Salud Publica Mex. 2011; 53 (Supl. 3): S375-85.

56. Organización de las Naciones Unidas para la Alimentación y la Agricultura. Una población sana depende de sistemas alimentarios saludables: Día Mundial de la Alimentación 16 de octubre de 2013. Roma: FAO; 2013.

57. International Zinc Nutrition Consultative Group. Fortificação com zinco. Resumo Técnico n. 4; 2007.
58. Jaime PC, Silva ACF, Lima AMC, Bortolini GA. Ações de alimentação e nutrição na atenção básica: a experiência de organização no Governo Brasileiro. Rev Nutr. 2011; 24 (6): 809-24.

59. Araújo ACT, Campos JADB. Subsídios para a avaliação do estado nutricional de crianças e adolescentes por meio de indicadores antropométricos. Alim Nutr. 2008; 19 (2): 21925.

Recebido em 8 de agosto de 2013

Versão final apresentada em 10 de dezembro de 2013

Aprovado em 6 de janeiro de 2014 